\title{
Optimal Penney Ante Strategy via Correlation Polynomial Identities
}

\author{
Daniel Felix \\ Department of Mathematics \\ University of California at San Diego, La Jolla, CA 92093-0112 \\ dfelix@math.ucsd.edu
}

Submitted: Oct 19, 2004; Accepted: Mar 30 2006; Published: Apr 4, 2006

Mathematics Subject Classifications: 60C05, 05A19

\begin{abstract}
In the game of Penney Ante two players take turns publicly selecting two distinct words of length $n$ using letters from an alphabet $\Omega$ of size $q$. They roll a fair $q$ sided die having sides labelled with the elements of $\Omega$ until the last $n$ tosses agree with one player's word, and that player is declared the winner. For $n \geq 3$ the second player has a strategy which guarantees strictly better than even odds. Guibas and Odlyzko have shown that the last $n-1$ letters of the second player's optimal word agree with the initial $n-1$ letters of the first player's word. We offer a new proof of this result when $q \geq 3$ using correlation polynomial identities, and we complete the description of the second player's best strategy by characterizing the optimal leading letter. We also give a new proof of their conjecture that for $q=2$ this optimal strategy is unique, and we provide a generalization of this result to higher $q$.
\end{abstract}

\section{Introduction}

We are interested in a generalization of the coin flipping game Penney Ante, invented in 1969 by Walter Penney. In its original version, two players take turns publicly selecting distinct binary sequences of a fixed length $n$. They flip a fair coin with sides labelled 0 and 1 until the last $n$ results match one player's sequence, and that player is declared the winner.

We study a version of this game in which the coin is abandoned in favor of a fair $q$ sided die. The faces of our die are labelled with the elements of a set $\Omega$ of size $q$. We call $\Omega$ an alphabet, and its elements letters. We will refer to a finite string of letters as a word.

Penney Ante's most striking feature is the nontransitivity of its beating relation among words of fixed length $n \geq 3$, where we say the word $X$ beats the word $Y$ if it is more likely to appear first. This nontransitivity is intimately related to the correlation polynomial of 
two words, introduced by Leo Guibas and Andrew Odlyzko in a 1981 paper [3]. Strictly speaking, the correlation polynomial of two words is a generating function. Its coefficients indicate how these words can be combined into a single word in which all of the overlaps are consistent. We will denote the correlation polynomial of the words $X=x_{1} \ldots x_{n}$ and $Y=y_{1} \ldots y_{m}$ by $[X, Y]$, and it is defined as follows:

$$
[X, Y]=\sum_{i=0}^{n-1} f(n-i) z^{i},
$$

where $f(i)$ is the indicator function

$$
f(i)= \begin{cases}1 & \text { if the word } x_{i} \ldots x_{n} \text { is a prefix of the word } y_{1} \ldots y_{m} \\ 0 & \text { otherwise. }\end{cases}
$$

In words, if $m=n$, the $z^{k}$ coefficient of $[X, Y]$ is 1 if the final $k+1$ letters of $X$ agree with the initial $k+1$ letters of $Y$. All other coefficients are zero, including those for which $k+1>n$. In general, $[X, Y] \neq[Y, X]$, as the former polynomial describes how $X$ and $Y$ can be combined with $X$ coming first, while the latter describes how they can be combined with $Y$ coming first. We will make frequent use of the evaluation of such a polynomial at $z=q$, so we denote this evaluation by $[X, Y]_{q}$. Our notation is a slight departure from [3], as we reserve juxtaposition for concatenation.

Throughout this paper we let $\Omega=\left\{\omega_{1} \ldots \omega_{q}\right\}$, using $\omega$ to denote an arbitrary letter in $\Omega$. We let $A=a_{1} \ldots a_{n}$ be the fixed word selected by the first player, defining $A^{\prime}$ to be the word $a_{1} \ldots a_{n-1}$ consisting of the first $n-1$ letters of $A$. Additionally, we denote the concatenations $A^{\prime} \omega_{i}$ by $A_{i}$, with the convention that $A_{1}=A$.

We define a period of a word $X=x_{1} \ldots x_{n}$ to be any nonnegative integer $\rho$ for which $x_{1} \ldots x_{n-\rho}=x_{\rho+1} \ldots x_{n}$. We call 0 the trivial period, and we define the basic period of $X$ to be its smallest positive period, with the convention that the basic period is $n$ if $X$ has no nontrivial periods.

John Conway (see [2]) was the first to discover that the odds that a word $Y$ beats a word $X$ are given by the elegant expression

$$
\frac{[X, X]_{q}-[X, Y]_{q}}{[Y, Y]_{q}-[Y, X]_{q}},
$$

though his proof was never published. This formula is the cornerstone on which nearly all of the analysis of Penney Ante has been based. Li [4] gave a proof of this formula using martingale techniques, and Guibas and Odlyzko gave a short proof involving a nonsingular system of equations of generating functions. They also proved that if the first player chooses the word $a_{1} \ldots a_{n}$, then the second player's best strategy is to select a word of the form $b a_{1} \ldots a_{n-1}$ for some appropriate $b$. They remarked that a complete description of the optimal leading letter did not seem to be simple, and they went on to conjecture that for $q=2$ and $A$ fixed this optimal letter is unique

In this article we give a complete description of the second player's best strategy. Our description is based on establishing that an optimal leading letter is any one which 
minimizes

$$
F_{q}(\omega):=q\left[A, \omega A^{\prime}\right]_{q}+\sum_{i=2}^{q}\left[A_{i}, \omega A^{\prime}\right]_{q} .
$$

This fact is our main result. We also prove a generalized version of Guibas and Odlyzko's uniqueness conjecture, first shown to be true in its original form by János Csirik in 1992 [1]. Our approach uses a set of correlation polynomial identities which we provide in the next section.

\section{Correlation polynomial identities}

We begin by letting $X=x_{1} \ldots x_{n}$ and $Y=y_{1} \ldots y_{n}$ be two words of length $n \geq 1$. We use $\delta$ to denote the familiar characteristic function.

Theorem 2.1. The following set of equations always holds:

$$
\begin{aligned}
& \sum_{i=1}^{q}\left[X \omega_{i}, \omega_{j} Y\right]=z\left[\omega_{j} X, \omega_{j} Y\right]-z^{n+1} \delta(X=Y)+1 \\
& \sum_{j=1}^{q}\left[X \omega_{i}, \omega_{j} Y\right]=z\left[X \omega_{i}, Y \omega_{i}\right]-z^{n+1} \delta(X=Y)+1 \\
& \sum_{i=1}^{q}\left[X \omega_{i}, Y \omega_{i}\right]=\sum_{j=1}^{q}\left[\omega_{j} X, \omega_{j} Y\right] \\
& \sum_{j=1}^{q}\left[\omega_{j} X, \omega_{j} X\right]=z[X, X]+(q-1) z^{n}+1 \quad \text { for } n \geq 2 .
\end{aligned}
$$

Proof. The proofs are straightforward. To show (2), let $\left[X, \omega_{j} Y\right]=\sum_{k} c_{k} z^{k}$. Then

$$
\begin{aligned}
\sum_{i=1}^{q}\left[X \omega_{i}, \omega_{j} Y\right] & =\sum_{i=1}^{q}\left(\delta\left(\omega_{i}=\omega_{j}\right)+\sum_{k=0}^{n-1} c_{k} \delta\left(\omega_{i}=y_{k+1}\right) z^{k+1}\right) \\
& =1+\sum_{k=0}^{n-1} c_{k} z^{k+1} \\
& =1+z\left(\left[\omega_{j} X, \omega_{j} Y\right]-z^{n} \delta(X=Y)\right)
\end{aligned}
$$

and the result follows. Identity (3) follows from (2) and the observation that for any two words $u_{1} \ldots u_{n}$ and $v_{1} \ldots v_{n}$ we have $\left[u_{1} \ldots u_{n}, v_{1} \ldots v_{n}\right]=\left[v_{n} \ldots v_{1}, u_{n} \ldots u_{1}\right]$. One can 
prove (4) by summing (2) over $j$ and (3) over $i$ and equating the results. Lastly,

$$
\begin{aligned}
\sum_{j=1}^{q}\left[\omega_{j} X, \omega_{j} X\right] & =\sum_{j=1}^{q}\left(z^{n}+\left[X, \omega_{j} X\right]\right) \\
& =\sum_{j=1}^{q}\left(z^{n}+\left[x_{1} \ldots x_{n}, \omega_{j} x_{1} \ldots x_{n-1}\right]\right) \\
& =q z^{n}+z[X, X]-z^{n}+1,
\end{aligned}
$$

proving (5). In the last step we have used (3) with $X=Y=x_{1} \ldots x_{n-1}$ and $\omega_{i}=x_{n}$. This substitution requires the word $x_{1} \ldots x_{n-1}$ to be nonempty, justifying the caveat $n \geq 2$.

Generalizations of these equations do exist; we confine ourselves to the above list since we will need nothing stronger. For example, (4) and (5) can be applied inductively to produce identities involving sums taken over all words of any fixed length.

\section{A lower bound and the $q=2$ case}

In this section we return to the game of Penney Ante played with a fair $q$-sided die. We shall only concern ourselves with words of length $n \geq 3$, for otherwise the game displays no intransitivity. Guibas and Odlyzko have shown that the second player's optimal word must be a concatenation of the form $b A^{\prime}$ for some appropriate letter $b$. We denote the words of this form by $B_{1}, \ldots, B_{q}$, with the convention that, among all these words, $B_{1}$ performs the best against $A$

As we have seen, the odds that $B_{i}$ will beat $A$ is given by Conway's formula:

$$
\frac{[A, A]_{q}-\left[A, B_{i}\right]_{q}}{\left[B_{i}, B_{i}\right]_{q}-\left[B_{i}, A\right]_{q}}
$$

The second player is not allowed to select the word $A$, so the above numerator and denominator are both positive for all $i$. Hence, since the odds that $B_{i}$ will beat $A$ are maximized for $i=1$,

$$
\frac{[A, A]_{q}-\left[A, B_{1}\right]_{q}}{\left[B_{1}, B_{1}\right]_{q}-\left[B_{1}, A\right]_{q}} \geq \frac{\sum_{i=1}^{q}\left([A, A]_{q}-\left[A, B_{i}\right]_{q}\right)}{\sum_{i=1}^{q}\left(\left[B_{i}, B_{i}\right]_{q}-\left[B_{i}, A\right]_{q}\right)} .
$$

This inequality holds because the right hand side is nothing more than a weighted average of the odds in favor of the $B_{i}$ 's, with $B_{i} \neq A$ having weight

$$
\frac{\left[B_{i}, B_{i}\right]_{q}-\left[B_{i}, A\right]_{q}}{\sum_{j=1}^{q}\left(\left[B_{j}, B_{j}\right]_{q}-\left[B_{j}, A\right]_{q}\right)}
$$


If $A$ is not an $n$-fold repetition of a single letter, $\left[B_{i}, A\right]=\left[A^{\prime}, A^{\prime}\right]$ for all $i$. We use this fact, together with identities (3) and (5), to obtain from (6) that

$$
\begin{aligned}
\frac{[A, A]_{q}-\left[A, B_{1}\right]_{q}}{\left[B_{1}, B_{1}\right]_{q}-\left[B_{1}, A\right]_{q}} & \geq\left.\frac{q[A, A]-z[A, A]+z^{n}-1}{z\left[A^{\prime}, A^{\prime}\right]+(q-1) z^{n-1}+1-q\left[A^{\prime}, A^{\prime}\right]}\right|_{z=q} \\
& =\frac{q^{n}-1}{q^{n}-q^{n-1}+1} \\
& =\frac{q}{q-1}-\frac{2 q-1}{(q-1)\left(q^{n}-q^{n-1}+1\right)} .
\end{aligned}
$$

This inequality also holds when $A$ is an $n$-fold repetition of a single letter $a$, since then the word $B_{1}=b a \ldots a$, with $b \neq a$, wins with even better odds. The odds that $B_{1}$ wins are therefore at least $q /(q-1)-O\left(q^{-n}\right)$ as $n \rightarrow \infty$, an improvement over the lower bound $q /(q-1)-O\left(q^{-n / 2}\right)$ found by Guibas and Odlyzko. Our bound is not sharp, and in fact Csirik gives the first player's optimal strategy for $q=2$ [1]. He finds that in a well-played game the second player's best odds are $\left(2^{n-1}+1\right) /\left(2^{n-2}+1\right)$, a figure whose deviation from 2 tends to $2 / 3$ of that of $(7)$, as $n \rightarrow \infty$. So our lower bound is nearly tight in this case. Note that for $A=b a \ldots a, b \neq a$, the second player cannot achieve better odds than $q /(q-1)$.

For $q \geq 2$ and $n \geq 3$ the above odds are strictly greater than 1 , so this inequality constitutes a proof of Penney Ante's nontransitivity for these interesting cases. We would also like to point out that our use of (5) demands $\left|A^{\prime}\right| \geq 2$, so these results hold only when $n \geq 3$.

When $q \geq 3$, this bound leads to a simplified proof of Guibas and Odlyzko's result on the the form of the second player's optimal word. Choosing an n-fold repetition of a single letter $a$ is a terrible strategy for the first player, as his opponent simply chooses the word $B=b a \ldots a$, with $b \neq a$. This choice optimizes the numerator and denominator in Conway's formula simultaneously, plainly yielding the best odds that the second player can hope for. If the first player employs some other strategy, the basic period of $A$ will be at least 2 . It follows that $[A, A]_{q}$ will be at most $q^{n-1}+q^{n-3}+\cdots+1$. Hence

$$
\frac{[A, A]_{q}-[A, B]_{q}}{[B, B]_{q}-[B, A]_{q}} \leq \frac{q^{n-1}+\left(q^{n-2}-1\right) /(q-1)}{q^{n-1}-[B, A]_{q}} .
$$

Suppose the second player selects a word $B$ not of the form $b A^{\prime}$. Then $[B, A]_{q} \leq q^{n-3}+$ $\cdots+1$, so that

$$
\frac{[A, A]_{q}-[A, B]_{q}}{[B, B]_{q}-[B, A]_{q}} \leq \frac{q^{n-1}+\left(q^{n-2}-1\right) /(q-1)}{q^{n-1}-\left(q^{n-2}-1\right) /(q-1)} .
$$

Rearrangements show the expression on the right hand side is strictly less than $\left(q^{n}-\right.$ 1) $/\left(q^{n}-q^{n-1}+1\right)$ for all $q$ and $n$ considered. Therefore, no word which is not of the form $\omega A^{\prime}$ can deliver better odds for the second player than all those words having such a form.

We now consider the consequences of inequality (6) when $q=2$, although we will exercise some foresight and leave $q$ as a variable in our work. For $q=2$, the second player 
knows that her best choice is one of the two possibilities $B_{1}$ and $B_{2}$. Clearly, $B_{1}$ offers better odds if and only if

$$
\frac{[A, A]_{q}-\left[A, B_{1}\right]_{q}}{\left[B_{1}, B_{1}\right]_{q}-\left[B_{1}, A\right]_{q}} \geq t \frac{[A, A]_{q}-\left[A, B_{1}\right]_{q}}{\left[B_{1}, B_{1}\right]_{q}-\left[B_{1}, A\right]_{q}}+(1-t) \frac{[A, A]_{q}-\left[A, B_{2}\right]_{q}}{\left[B_{2}, B_{2}\right]_{q}-\left[B_{2}, A\right]_{q}}
$$

for any fixed $t \in[0,1)$. The right hand side of (6) is precisely of this form, so inequality (6) is actually equivalent to the statement that $B_{1}$ is an optimal choice for the second player. Rearrangement of (7) then yields that $B=b A^{\prime}$ is optimal if and only if

$$
[A, B]_{q}-[A, A]_{q}+\left(\frac{q}{q-1}-\alpha\right)\left([B, B]_{q}-[B, A]_{q}\right) \leq 0,
$$

where $\alpha$ is the positive term

$$
\frac{2 q-1}{(q-1)\left(q^{n}-q^{n-1}+1\right)}
$$

of order $q^{-n}$ in (7). Since $q=2, b$ is optimal if and only if the left hand side of the above inequality is minimized. Removing constants and those terms dependent only on $A$, such as $[B, A]_{q}=\left[A^{\prime}, A^{\prime}\right]_{q}$, we find that $b$ is optimal precisely when

$$
\begin{aligned}
& {[A, B]_{q}+\left(\frac{q}{q-1}-\alpha\right)[B, B]_{q} } \\
= & {[A, B]_{q}+\left(\frac{q}{q-1}-\alpha\right)\left(\frac{1}{q}\right)\left(q^{n}-1+\sum_{i=1}^{q}\left[A_{i}, B\right]_{q}\right) }
\end{aligned}
$$

is minimized, with equality holding by (2). Recall that $A_{i}$ is defined to be the concatenation $A^{\prime} \omega_{i}$. Dropping constant terms and dividing out by the positive constant preceding the above sum presents us with the equivalent problem of minimizing

$$
\left(q+\frac{(q-1)^{2} \alpha}{q-(q-1) \alpha}\right)[A, B]_{q}+\sum_{i=2}^{q}\left[A_{i}, B\right]_{q}
$$

The $q=2$ case of our main result now begins to take shape.

Theorem 3.1. For $q=2$, the odds in favor of $B=b A^{\prime}$ are maximized precisely for those letters which minimize

$$
F_{2}(\omega):=2\left[A, \omega A^{\prime}\right]_{2}+\left[A_{2}, \omega A^{\prime}\right]_{2} .
$$

Conway's formula tells us that an optimal choice of $b$ will necessarily make both $[A, B]_{2}$ and $[B, B]_{2}$ small, though it is unclear exactly how we should proceed if these cannot be simultaneously minimized. Theorem 3.1 shows that the solution simply involves minimizing a linear combination of correlation polynomials evaluated at 2 . 
Proof. We temporarily assume that $A$ does not consist of two alternating (though not necessarily distinct) letters, leaving this case to be treated separately. This mild restriction ensures that $[A, B]$ has degree at most $n-3$. Hence $[A, B]_{2} \leq 2^{n-3}+\cdots+1$ so that

$$
\begin{aligned}
\frac{(2-1)^{2} \alpha}{2-(2-1) \alpha}[A, B]_{2} & \leq \frac{(2-1) \alpha}{2-(2-1) \alpha}\left(2^{n-2}-1\right) \\
& =\frac{3\left(2^{n-2}-1\right)}{2^{n+1}-2^{n}-2+1}
\end{aligned}
$$

which is strictly less than 1.

We now assume that $b_{1}$ minimizes $F_{2}(\omega)$ and consider $b_{2}$ which minimizes (8), letting $B_{1}$ and $B_{2}$ denote the concatenations $b_{1} A^{\prime}$ and $b_{2} A^{\prime}$, respectively. If $b_{2}$ does not minimize $F_{2}(\omega)$, then, because this expression is always an integer,

$$
\begin{aligned}
2\left[A, B_{2}\right]_{2}+\left[A_{2}, B_{2}\right]_{2} & \geq 1+2\left[A, B_{1}\right]_{2}+\left[A_{2}, B_{1}\right]_{2} \\
& >\left(2+\frac{(2-1)^{2} \alpha}{2-(2-1) \alpha}\right)\left[A, B_{1}\right]_{2}+\left[A_{2}, B_{1}\right]_{2}
\end{aligned}
$$

so that $b_{2}$ cannot possibly minimize (8). Hence every letter which minimizes (8) must also minimize $F_{2}(\omega)$.

The reverse containment also holds, for consider two distinct letters $b_{1}$ and $b_{2}$ which minimize $F_{2}(\omega)$. Then

$$
2\left[A, B_{1}\right]_{2}+\left[A_{2}, B_{1}\right]_{2}=2\left[A, B_{2}\right]_{2}+\left[A_{2}, B_{2}\right]_{2} .
$$

Taking this equation modulo 2 gives $b_{1}=b_{2}$, a contradiction. Thus $F_{2}(\omega)$ is uniquely minimized by the same letter which uniquely minimizes (8).

It only remains to check the case in which $A$ is composed of alternating letters. The best choice for the second player's leading letter is obvious in this case; one option will yield a string of alternating letters which, by symmetry, will beat $A$ with probability $1 / 2$. Since we know that the second player always has a choice delivering strictly better odds, it is clear that the optimal letter $b$ is the one satisfying $b=a_{1}$. A quick check shows that, for the suboptimal choice, the right hand side of (9) evaluates to at least $2^{n-1}$, while the correct choice evaluates to at most 3 depending on the parity of $n$. Hence our claim holds for all $n \geq 3$.

We have an immediate corollary, which follows upon taking (9) modulo 2 as in the above proof.

Corollary 3.1. For $q=2$, the second player's optimal choice is unique.

Additionally, we can now give a simple description of the correct leading letter $b$ of the second player's optimal choice $B=b A^{\prime}$.

Corollary 3.2. Let $r_{1}$ and $r_{2}$ be the basic periods of $A=A_{1}$ and $A_{2}$, respectively, and let $r=\min \left(r_{1}, r_{2}\right)$. Let $\Omega=\{0,1\}$, and define $\bar{\omega}:=1-\omega$. Then the optimal $b$ is given by

$$
b= \begin{cases}\bar{a}_{r+1} & \text { if } r_{1}=r_{2}+1 \text { and } r_{2} \leq n-2 \\ \bar{a}_{r} & \text { otherwise. }\end{cases}
$$


This description agrees in almost all cases with the approximation supplied by Guibas and Odlyzko, who found that one winning strategy for picking $b$ is given by

$$
b= \begin{cases}\bar{a}_{r} & \text { if } r \leq n-1 \\ \bar{a}_{n-1} & \text { if } r=n .\end{cases}
$$

With this winning strategy they were able to establish their lower bound for the odds in favor of the optimal $B$, as well as prove that an optimal selection must be a concatenation of the form $\omega A^{\prime}$.

Proof. We begin by letting $\pi\left(A_{i}\right)$ be the set of nontrivial periods of the word $A_{i}$, always including the period $n$ corresponding to the overlap of length 0 . By the definition of the correlation polynomial, we may rewrite (9) in the form

$$
F_{2}(b)=2\left(\sum_{\rho \in \pi(A)} \delta\left(b=a_{\rho}\right) 2^{n-\rho}\right)+\sum_{\rho \in \pi\left(A_{2}\right)} \delta\left(b=a_{(2, \rho)}\right) 2^{n-\rho},
$$

where $a_{(i, \rho)}$ denotes the $\rho$ th letter of the word $A_{i}$. We will suppress this notation when $i=1$ and when $\rho<n$, for then $a_{(i, \rho)}=a_{\rho}$.

Let $r, r_{1}$, and $r_{2}$ be defined as above and first consider the case $r_{1}=r_{2}$. Noticing that $\pi\left(A_{1}\right) \cap \pi\left(A_{2}\right)=\{n\}$, we must have $r_{1}=r_{2}=n$. Thus $F_{2}(b)=2 \delta\left(b=a_{n}\right)+\delta\left(b=\bar{a}_{n}\right)$, so that $b=\bar{a}_{n}$ is the correct choice, agreeing with our claim.

Suppose that $r=r_{1}<r_{2}$. Then (10) gives us the bounds $F_{2}\left(a_{r}\right) \geq 2\left(2^{n-r}\right)$ and $F_{2}\left(\bar{a}_{r}\right) \leq 2\left(2^{n-r-1}+\cdots+1\right)=2^{n-r+1}-2$. Thus $b=\bar{a}_{r}$ is the optimal choice since it minimizes $F_{2}(\omega)$.

The analysis is similar when $r_{1} \geq r_{2}+2$. We again use (10) to find $F_{2}\left(a_{r}\right)=F_{2}\left(a_{r_{2}}\right) \geq$ $2^{n-r}$ while $F_{2}\left(\bar{a}_{r}\right) \leq 2\left(2^{n-r-2}+2^{n-r-3}+\cdots+1\right)=2^{n-r}-2$. Hence $b=\bar{a}_{r}$ is again the optimal choice.

The only remaining case is $r_{1}=r_{2}+1$. For the moment we assume that $r_{2} \leq n-2$. As $r_{1}$ and $r_{2}$ are both periods, $a_{1} \ldots a_{n-r}=a_{r+1} \ldots a_{n-1} \bar{a}_{n}$ and $a_{1} \ldots a_{n-r-1}=a_{r+2} \ldots a_{n}$. Together, these imply $a_{r+1}=a_{r+2}=\cdots=a_{n}=a_{1}=a_{2}=\cdots=a_{n-r-1} \neq a_{n-r}$. In particular, this says that each of $r_{2}+1=r_{1}, r_{1}+1, \ldots n$ is a period of $A$. Thus, since $A$ and $A_{2}$ can only share the nontrivial period $n$,

$$
\begin{aligned}
\pi(A) & =\{r+1, r+2, \ldots, n\} \quad \text { and } \\
\pi\left(A_{2}\right) & =\{r, n\} .
\end{aligned}
$$

Therefore, since the final $n-r$ letters as well as the first $n-r-1$ letters of $A$ are all identical, $F_{2}\left(a_{r+1}\right) \geq 2\left(2^{n-r-1}+\cdots+1\right)=2^{n-r+1}-2$. Meanwhile, $F_{2}\left(\bar{a}_{r+1}\right) \leq 2^{n-r}+1$ since no period of $A$ can contribute to this value. These bounds imply $F_{2}\left(a_{r+1}\right)>F_{2}\left(\bar{a}_{r+1}\right)$ when $n-r \geq 2$. Thus the optimal choice is $b=\bar{a}_{r+1}$, as long as $r \leq n-2$, as in the statement of the corollary. The final case is $r_{2}=n-1$ and $r_{1}=n$. Now $F_{2}\left(a_{n-1}\right) \geq 2$ since $n-1 \in \pi\left(A_{2}\right)$, but $F_{2}\left(\bar{a}_{n-1}\right) \leq 2$ because this choice prevents the first two letters of $B$ from being equal to the last two letters of either $A$ or $A_{2}$. We may never have $F_{2}(\omega)=F_{2}(\bar{\omega})$ (taking this equation modulo 2 produces a contradiction), so the correct choice is $b=\bar{a}_{n-1}=\bar{a}_{r}$, which is in accordance with our claim. 


\section{The general case}

In this section we establish that a version of (9) actually holds for all $q$, and we use this to characterize the leading letter of the second player's best strategy. Our treatment of the general case will not require anything beyond the elementary techniques we have already used, and in extending Corollary 3.2 to higher $q$ we will be forced to sacrifice none of the simplicity of the $q=2$ result. We begin with a useful lemma which, after proving our main result, can be seen as a generalization of Corollary 3.1. We continue to use the notation introduced in the last section. Additionally, recall that $F_{q}(\omega)$ is defined by $(1)$.

Lemma 4.1. If $F_{q}(\omega)$ is not uniquely minimized, then its minimum value is 1.

The converse is not true. Consider the alphabet $\Omega=\{0,1,2\}$, and let $A$ be the word 11011. Then $F_{3}(0)=28, F_{3}(1)=12$, and $F_{3}(2)=1$.

Proof. The claim is vacuously true for $q=2$ by Corollary 3.1. For $q \geq 3$ we actually show something stronger; if $b_{1}, b_{2} \in \Omega$ are distinct, then $F_{q}\left(b_{1}\right)=F_{q}\left(b_{2}\right)$ implies both these values are 1.

For any fixed $\omega$, the polynomial

$$
\sum_{i=2}^{q}\left[A_{i}, \omega A^{\prime}\right]=\sum_{i=2}^{q} \sum_{\rho \in \pi\left(A_{i}\right)} \delta\left(\omega=a_{(i, \rho)}\right) z^{n-\rho}
$$

has only 0 and 1 as coefficients; this is true for the nonconstant terms because $\pi\left(A_{i}\right) \cap$ $\pi\left(A_{j}\right)=\{n\}$ for $i \neq j$. The constant term is at most 1 since $\omega$ equals at most one of the distinct letters $a_{(2, n)}, \ldots, a_{(q, n)}$. Since the polynomial $\left[A, \omega A^{\prime}\right]$ has this same property, it follows that for any fixed $\omega$

$$
P_{\omega}(z):=z\left[A, \omega A^{\prime}\right]+\sum_{i=2}^{q}\left[A_{i}, \omega A^{\prime}\right]
$$

is a polynomial whose nonzero coefficients can only be 1 or 2 . Moreover, the coefficient of the $z^{n-\rho}$ term can be 2 only if $\omega=a_{(i, \rho)}$ and $\rho \in \pi\left(A_{i}\right)$ for some $i \geq 2$, while $\omega=a_{\rho+1}$ and $\rho+1 \in \pi(A)$.

Observe that $P_{\omega}(q)=F_{q}(\omega)$. Since each of the coefficients of $P$ is strictly less than $3 \leq q$, these coefficients are simply the digits of $F_{q}(\omega)$ expressed in base $q$. If $F_{q}\left(b_{1}\right)=$ $F_{q}\left(b_{2}\right)$, then clearly their base $q$ representations are equal so that $P_{b_{1}}(z)=P_{b_{2}}(z)$.

Let $B_{1}$ and $B_{2}$ be words $b_{1} A^{\prime}$ and $b_{2} A^{\prime}$, respectively, where $b_{1} \neq b_{2}$. Consider the leading nonzero term $c_{k} z^{k}$ of the identical polynomials $P_{b_{1}}(z)$ and $P_{b_{2}}(z)$ (note $P_{\omega}(z)$ is clearly never the zero polynomial). The coefficient $c_{k}$ cannot be 2 , since this would imply that the leading terms of the $z\left[A, B_{1}\right]$ and $z\left[A, B_{2}\right]$ summands are both $z^{k}$, yielding the contradiction $b_{1}=b_{2}=a_{n-k+1}$. Similarly, the leading terms of the $\sum_{i}\left[A_{i}, B_{1}\right]$ and $\sum_{i}\left[A_{i}, B_{2}\right]$ summands cannot both be $z^{k}$ for some $k \geq 1$, as this implies $b_{1}=b_{2}=a_{n-k}$.

Assuming that $k \geq 1$, we therefore have, without loss of generality, that the leading term of $P_{b_{1}}(z)$ is the leading term of $z\left[A, B_{1}\right]$, while that of $P_{b_{2}}(z)$ comes from $\sum_{i}\left[A_{i}, B_{2}\right]$. 
In terms of word overlaps this gives that $b_{1} a_{1} \ldots a_{k-1}=a_{n-k+1} \ldots a_{n}$ and $b_{2} a_{1} \ldots a_{k}=$ $a_{n-k} \ldots a_{n-1} a_{(i, n)}$ for some $i \geq 2$. In particular, as in the proof of Corollary $3.2, b_{1}=a_{1}=$ $\cdots=a_{k-1}=a_{n-k+1}=\cdots=a_{n} \neq a_{k}$. Thus the first $k$ letters of $B_{1}$ are equal to the last $k$ letters of $A$ so that $F_{q}\left(b_{1}\right)=P_{b_{1}}(q)=q^{k}+\cdots+q \equiv 0 \bmod q$. But $b_{2} \neq b_{1}=a_{n}$, implying $F_{q}\left(b_{2}\right) \equiv 1 \bmod q$, another contradiction. It must then be the case that $k=0$, and the lemma follows immediately.

We are now ready to prove our main result.

Theorem 4.1. The odds in favor of $B=b A^{\prime}$ are maximized precisely for those $b$ which minimize

$$
F_{q}(\omega)=q\left[A, \omega A^{\prime}\right]_{q}+\sum_{i=2}^{q}\left[A_{i}, \omega A^{\prime}\right]_{q} .
$$

Proof. The proof is divided into several parts according to how well the second player can perform against a fixed $A$.

We first consider the case in which no word can deliver odds better than $q /(q-1)$ in favor of the second player. Using the lower bound provided by (6), the best beater of $A$ wins with odds of $q /(q-1)-t \alpha$ for some $t$ contained in the closed interval $[0,1]$. Thus $b$ is optimal if and only if

$$
\frac{[A, A]_{q}-[A, B]_{q}}{[B, B]_{q}-[B, A]_{q}} \geq \frac{q}{q-1}-t \alpha
$$

Proceeding as in the derivation of (8), we find that maximizing the odds in favor of $B$ is equivalent to minimizing

$$
\left(q+\frac{(q-1)^{2} t \alpha}{q-(q-1) t \alpha}\right)[A, B]_{q}+\sum_{i=2}^{q}\left[A_{i}, B\right]_{q} .
$$

But $[A, B]_{q} \leq q^{n-2}+\cdots+1=\left(q^{n-1}-1\right) /(q-1)$ because $A \neq B$, giving

$$
\begin{aligned}
\frac{(q-1)^{2} t \alpha}{q-(q-1) t \alpha}[A, B]_{q} & \leq \frac{(q-1) t \alpha}{q-(q-1) t \alpha}\left(q^{n-1}-1\right) \\
& \leq \frac{2 q^{n}-q^{n-1}-2 q+1}{q^{n+1}-q^{n}-q+1}
\end{aligned}
$$

The second inequality holds because the right hand side of (14) is an increasing function of $t$ on the interval $[0,1]$. The resulting upper bound is strictly less than 1 when $q \geq 3$ (it is false for $q=2$, necessitating the slightly different argument in Theorem 3.1). Just as in the proof of Theorem 3.1, this strict upper bound implies that every letter which minimizes (13) also minimizes $F_{q}(\omega)$. We must now prove that the converse also holds. This is obvious if $F_{q}(\omega)$ is uniquely minimized. Otherwise, if many letters minimize $F_{q}(\omega)$, this minimum value must be 1 by Lemma 4.1 . Hence $\left[A, \omega A^{\prime}\right]_{q}$ must be zero for each of these letters. It follows that, when evaluated at some $b_{0}$ which minimizes $F_{q}(\omega)$, expression (13) is equal to $F_{q}\left(b_{0}\right)=1$. This is clearly its minimum, which proves our claim for this case. 
We now consider the case in which the second player can achieve odds strictly better than $q /(q-1)$, making the simplifying assumption that $A^{\prime}$ does not consist of one repeated letter. This will put a manageable upper bound on the odds in favor of the second player, and the cases we have left out will be easily dealt with later. With this assumption, the basic period of $A^{\prime}$ will be at least 2 , and $\left[a_{2} \ldots a_{n}, A^{\prime}\right]_{q} \leq q^{n-3}+\cdots+1$. Hence,

$$
\begin{aligned}
\frac{[A, A]_{q}-\left[A, B_{i}\right]_{q}}{\left[B_{i}, B_{i}\right]_{q}-\left[B_{i}, A\right]_{q}} & =\frac{q^{n-1}+\left[a_{2} \ldots a_{n}, A^{\prime}\right]_{q}-[A, B]_{q}}{q^{n-1}+\left[A^{\prime}, B\right]_{q}-\left[A^{\prime}, A^{\prime}\right]_{q}} \\
& \leq \frac{q^{n-1}+\left[a_{2} \ldots a_{n}, A^{\prime}\right]_{q}}{q^{n-1}-\left[A^{\prime}, A^{\prime}\right]_{q}} \\
& \leq \frac{q^{n-1}+\left(q^{n-2}-1\right) /(q-1)}{q^{n-1}-q^{n-2}-\left(q^{n-3}-1\right) /(q-1)}
\end{aligned}
$$

so that the second player never has odds better than

$$
\frac{q}{q-1}+\frac{q^{n-1}-2 q+1}{(q-1)\left(q^{n}-2 q^{n-1}+q^{n-2}-q^{n-3}+1\right)} .
$$

Letting $\beta$ be the term of order $q^{-2}$ on the right hand side, we may then write her best odds as

$$
\frac{q}{q-1}+t \beta
$$

where $t$ is a real parameter contained in the interval $(0,1]$. Using the argument giving (8) with $\alpha \mapsto-t \beta$, the second player achieves her maximal odds when $b$ minimizes

$$
H_{q}(\omega):=\left(q-\frac{(q-1)^{2} t \beta}{q+(q-1) t \beta}\right)\left[A, \omega A^{\prime}\right]_{q}+\sum_{i=2}^{q}\left[A_{i}, \omega A^{\prime}\right]_{q} .
$$

For notational convenience, let $\gamma$ be given by

$$
\gamma=\frac{(q-1)^{2} t \beta}{q+(q-1) t \beta}
$$

Since $\gamma$ is an increasing function of $t$ for $t \in(0,1]$,

$$
\gamma \leq\left.\gamma\right|_{t=1}<\frac{q-1}{q+1}
$$

The second inequality above can be verified by showing $\beta \leq(q-1)^{-1}$, though we spare the reader the details.

We continue by describing the letters which minimize $F_{q}(\omega)$ and $H_{q}(\omega)$, showing that these sets are necessarily identical. This is immediate if $F_{q}(\omega)$ is not uniquely minimized. For then, by Lemma 4.1 , its minimum value is 1 and $\left[A, b_{0} A^{\prime}\right]_{q}=0$ for any $b_{0}$ satisfying $F_{q}\left(b_{0}\right)=1$. This in turn forces $H_{q}\left(b_{0}\right)=1$, and this is clearly its minimum. Additionally, $H_{q}\left(b_{0}\right)=1$ implies $F_{q}\left(b_{0}\right)=1$, so the sets of minimizing letters are identical. This line 
of reasoning actually shows slightly more; if the minimum value of $F_{q}(\omega)$ is 1 , then our claim is true. From now on, we may therefore assume that $F_{q}(\omega)$ is uniquely minimized by $b_{1}$, and that $F_{q}\left(b_{1}\right) \geq 2$.

We first consider the problem of minimizing $F_{q}(\omega)$. We know

$$
\begin{aligned}
F_{q}(\omega) & =q\left[A, \omega A^{\prime}\right]_{q}+\sum_{i=2}^{q} \sum_{\rho \in \pi\left(A_{i}\right)} \delta\left(\omega=a_{(i, \rho)}\right) q^{n-\rho} \\
& =\sum_{\rho \in \pi(A)} \delta\left(\omega=a_{\rho}\right) q^{n-\rho+1}+\sum_{i=2}^{q} \sum_{\rho \in \pi\left(A_{i}\right)} \delta\left(\omega=a_{(i, \rho)}\right) q^{n-\rho} .
\end{aligned}
$$

Let $\pi^{\prime}$ be the union $\cup_{i=2}^{q} \pi\left(A_{i}\right)$. Since $\pi\left(A_{i}\right) \cap \pi\left(A_{j}\right)=\{n\}$ for all $i \neq j$, and since distinct $A_{i}$ only differ in their final letters, we may rewrite the above as

$$
\sum_{\rho \in \pi(A)} \delta\left(\omega=a_{\rho}\right) q^{n-\rho+1}+\sum_{\rho \in \pi^{\prime} \backslash\{n\}} \delta\left(\omega=a_{\rho}\right) q^{n-\rho}+\delta\left(\omega \neq a_{n}\right) .
$$

Before proceeding, it will be convenient to let $\pi=\cup_{i=1}^{q} \pi\left(A_{i}\right)$, and to introduce the polynomial

$$
Q_{\omega}(z):=\sum_{i=1}^{q}\left[A_{i}, \omega A^{\prime}\right]=1+\sum_{\rho \in \pi \backslash\{n\}} \delta\left(\omega=a_{\rho}\right) z^{n-\rho} .
$$

Let $\tilde{b} \in \Omega$ be the letter which minimizes the degree of this polynomial. Call this minimum degree $d$, so that $\tilde{b}=a_{n-d}$, and let $\tilde{B}=\tilde{b} A^{\prime}$. Notice that the nonzero coefficients of $Q$ are always 1. Also, a direct comparison with (12) gives

$$
Q_{\omega}(q) \leq F_{q}(\omega) \leq q Q_{\omega}(q)
$$

Suppose that $b_{1} \neq \tilde{b}$. This forces $\operatorname{deg} Q_{b_{1}}(z)>d$; they cannot both be zero because this would imply $F_{q}\left(b_{1}\right)=1$, and they cannot both be identical and positive because this would imply $b_{1}=\tilde{b}=a_{n-d}$. Moreover, $\operatorname{deg} Q_{b_{1}}(z)$ cannot exceed $d+1$. If so,

$$
\begin{aligned}
F_{q}\left(b_{1}\right)-F_{q}(\tilde{b}) & \geq Q_{b_{1}}(q)-q Q_{\tilde{b}}(q) \\
& \geq q^{d+2}-q\left(q^{d}+\cdots+1\right) .
\end{aligned}
$$

This lower bound is at least 1 for $q \geq 2$, contradicting the minimality of $F_{q}\left(b_{1}\right)$.

Hence $\operatorname{deg} Q_{b_{1}}(z)=d+1$ if $b_{1} \neq \tilde{b}$, but more can be said before we investigate what consequences this has in terms of word overlaps. The leading term of $Q_{b_{1}}(z)$ cannot come from a period in $\pi(A)$, as this would imply

$$
F_{q}\left(b_{1}\right)-F_{q}(\tilde{b}) \geq q\left(q^{d+1}\right)-q Q_{\tilde{b}}(q)
$$

and the contradiction follows exactly as above. Similarly, the leading term of $Q_{\tilde{b}}(z)$ must come from a period in $\pi(A)$, for otherwise

$$
F_{q}\left(b_{1}\right)-F_{q}(\tilde{b}) \geq q^{d+1}-\left(q^{d}+q\left(q^{d-1}+\cdots+1\right)\right)
$$


which is positive for $q \geq 3$, again contradicting the minimality of $F_{q}\left(b_{1}\right)$. Therefore, the leading term of $Q_{\tilde{b}}(z)$ must come from a period in $\pi(A)$, while the leading term of $Q_{b_{1}}(z)$ cannot. Equivalently, $n-d \in \pi(A)$ and $n-d-1 \in \pi\left(A_{l}\right)$ for some $l \geq 2$. In terms of word overlaps, $\tilde{b} a_{1} \ldots a_{d}=a_{n-d} \ldots a_{n}$ and $b_{1} a_{1} \ldots a_{d+1}=a_{n-d-1} \ldots a_{n-1} a_{(l, n)}$. Thus, $\tilde{b}=a_{1}=\cdots=a_{d}=a_{n-d}=\cdots=a_{n}$, yielding that all of $n-d+1, \ldots, n$ are periods of $A$. This gives us the exact forms of the $Q$ polynomials, since $\pi\left(A_{i}\right) \cap \pi\left(A_{j}\right)=\{n\}$ for $i \neq j$ :

$$
\begin{aligned}
Q_{\tilde{b}}(z) & =[A, \tilde{B}]=z^{d}+\cdots+1 \\
Q_{b_{1}}(z) & =\left[A_{l}, B_{1}\right]=z^{d+1}+1 .
\end{aligned}
$$

It follows that $F_{q}\left(b_{1}\right)=q^{d+1}+1$ and $F_{q}(\tilde{b})=q\left(q^{d}+\cdots+1\right)$. Hence $F_{q}\left(b_{1}\right)<F_{q}(\tilde{b})$ for all $d \geq 1$, so that the above description of $F_{q}\left(b_{1}\right)$ is minimal for these $d$. Otherwise, $b_{1}=\tilde{b}$.

To summarize, we have shown that if $\tilde{b}$ is the letter minimizing $d=\operatorname{deg} Q_{\omega}(z)$, and if $F_{q}(\omega)$ has minimum value greater than 1 , then $F_{q}(\omega)$ is minimized by the letter $b_{1}$ given by

$$
b_{1}= \begin{cases}a_{n-d-1} & \text { if } n-d \in \pi(A), n-d-1 \in \pi\left(A_{i}\right) \text { for some } i \geq 2, \\ & \operatorname{deg} Q_{a_{n-d-1}}(z)=d+1, \text { and } d \neq 0 \\ a_{n-d} & \text { otherwise. }\end{cases}
$$

If $F_{q}(\omega)$ has a minimum value of 1 , then $b_{1}$ can be chosen arbitrarily from the set of letters which give $F_{q}(\omega)=1$.

We must now repeat this argument to describe the letter $b_{2}$ which minimizes $H_{q}(\omega)$. If $b_{2} \neq \tilde{b}$, then we again have $\operatorname{deg} Q_{b_{2}}(z)>d$ by the same reasoning as above. Additionally, the above proof that $\operatorname{deg} Q_{b_{2}}(z)<d+2$ can be used again, since

$$
q^{d+2}-(q-\gamma)\left(q^{d}+\cdots+1\right)>q^{d+2}-q\left(q^{d}+\cdots+1\right)>0
$$

for all positive $\gamma$. Hence $\operatorname{deg} Q_{b_{2}}(z)=d+1$.

It must also be true that $n-d-1 \notin \pi(A)$, since then

$$
H_{q}\left(b_{2}\right)-H_{q}(\tilde{b}) \geq(q-\gamma)\left(q^{d+1}\right)-(q-\gamma)\left(q^{d}+\cdots+1\right),
$$

which is strictly positive for our $q$ and $\gamma$, contradicting the minimality of $H_{q}\left(b_{2}\right)$. Similarly, it must also be the case that $n-d \in \pi(A)$, for otherwise

$$
H_{q}\left(b_{2}\right)-H_{q}(\tilde{b}) \geq q^{d+1}-\left(q^{d}+(q-\gamma)\left(q^{d}+\cdots+1\right)\right)
$$

and right hand side is positive for all $q \geq 3$.

Given that $n-d \in \pi(A)$ and $n-d-1 \in \pi\left(A_{l}\right)$ for some $l \geq 2$, our $Q$ polynomials have exactly the same form as in the previous argument. Hence $H_{q}\left(b_{2}\right)<H_{q}(\tilde{b})$ (so that $b_{2}$ truly minimizes $H$ ) precisely when

$$
q^{d+1}+1<(q-\gamma) \frac{q^{d+1}-1}{q-1}
$$


Use of the upper bound $\gamma<(q-1) /(q+1)$ shows that this holds for all $d \geq 1$, agreeing with the description of $b_{1}$.

To finish the proof of our theorem we need only resolve the case in which $A^{\prime}$ consists of a single repeated letter. In fact, we have already solved it, for in this restrictive case $A$ itself can be composed of at most two distinct letters. Since $q \geq 3$, this gives the second player the option of beginning her word with a third previously unused letter $b_{3}$. Clearly $[A, B]_{q}$ and $\left[A^{\prime}, B\right]_{q}$ will both be simultaneously minimized by $b_{3}$ in this case, each taking the value zero. It follows that $F_{q}\left(b_{3}\right)=1$. Our earlier treatment of this case did not rely on any assumptions about the form of $A^{\prime}$, and this observation completes the proof.

This description of the optimal leading letter does agree with the $q=2$ description given earlier. We chose to state the $q=2$ result differently to highlight its similarity with Guibas and Odlyzko's approximation.

Our use of the polynomial $Q_{\omega}(z)$ does not detract from the simplicity of the result, for the letter which minimizes its degree is precisely the letter which maximizes the basic period of $\omega A^{\prime}$. We integrate this description with the proof of our main result to provide an equivalent description of the second player's optimal leading letter which avoids any mention of $Q_{\omega}(z)$.

Corollary 4.1. Let $A=a_{1} \ldots a_{n}$ be the word selected by the first player, and suppose $\pi=\left\{p_{1}, \ldots, p_{k}\right\}$, where $p_{1} \leq \cdots \leq p_{k}=n$. Supposing that such a period exists, let $p_{j}$ be the smallest element of $\pi$ such that

$$
\bigcup_{i=1}^{j}\left\{a_{p_{i}}\right\}=\Omega .
$$

Then the letter $b$ which maximizes the second player's odds of winning is given by

$$
b= \begin{cases}a_{p_{j}-1} & \text { if } p_{j} \in \pi(A), p_{j}-1 \in \pi\left(A_{l}\right) \text { for some } l \geq 2, \\ & p_{j}-1=\min \left\{\rho \in \pi\left(A_{l}\right)\right\}, \text { and } p_{j} \neq n \\ a_{p_{j}} & \text { otherwise. }\end{cases}
$$

If no such $p_{j}$ exists, then $b$ can be chosen arbitrarily from $\Omega \backslash\left\{a_{p_{1}}, \ldots, a_{p_{k}}\right\}$.

Since $F_{q}(\omega)=1$ if and only if both $\left[A, \omega A^{\prime}\right]$ and $\left[A^{\prime}, \omega A^{\prime}\right]$ are zero, we have the following generalization of Corollary 3.1.

Corollary 4.2. If two distinct words maximize the second player's odds of winning, then these odds are

$$
\frac{[A, A]_{q}}{q^{n-1}-\left[A^{\prime}, A^{\prime}\right]_{q}}
$$

When $q=2$, the above odds cannot be achieved by any two distinct words, as in the proof of Corollary 3.1. Note that these odds are at least $q /(q-1)$. 


\section{Concluding remarks}

We conclude by admitting that while our main result is appealing, the piecemeal proof we provide is not entirely satisfying. It would be desirable to have a cleaner, more intuitive proof of Theorem 4.1. Unfortunately, it is not the case that the second player's optimal choice, even if unique, is the only one delivering odds better than $q /(q-1)$, as this would greatly simplify the proof. For example, let $\Omega=\{0,1,2\}$ and $A=001$. Then the word 100 beats $A$ with odds of $8 / 5$ while 200 wins with odds of $9 / 5$, both of which are greater than $3 / 2$.

It would also be of interest to find an analogs of Theorem 4.1 and Corollary 4.1 in the case of a biased die. This may be difficult since not every probability distribution on $\Omega$ will give rise to a nontransitive winning relation. Dudley Stark [6] has characterized all probability distributions having this property for all sufficiently long words.

\section{Acknowledgements}

The author would like to thank Fan Chung for her support during the writing of this article, and an anonymous referee for numerous helpful suggestions.

\section{References}

[1] J. A. Csirik, Optimal strategy for the first player in the penney ante game, Combinatorics, Probability, and Computing 1 (1992): 311-321.

[2] M. Gardner, On the paradoxical situations that arise from nontransitive situations, Scientific American (Oct 1974): 120-125.

[3] L. J. Guibas and A. M. Odlyzko, String overlaps, pattern matching, and nontransitive games, Journal of Combinatorial Theory (A) 30 (1981): 183-208.

[4] S.-Y. R. Li, A martingale approach to the study of occurrence of sequence patterns in repeated experiments, Annals of Probability 8 (Dec 1980): 1171-1176.

[5] W. Penney, Problem 95: penney-ante, Journal of Recreational Mathematics 2 (1969): 241.

[6] D. Stark, First occurrence in pairs of long words: a penney-ante conjecture of pevzner, Combinatorics, Probability, and Computing 4 (1995): 279-285. 